\title{
О РАЗРЕШИМОСТИ КОНЕЧНОЙ ГРУППЫ С ПОЛУНОРМАЛЬНЫМИ ИЛИ СУБНОРМАЛЬНЫМИ ПОДГРУППАМИ ШМИДТА НЕКОТОРОЙ ЕЕ МАКСИМАЛЬНОЙ ПОДГРУППЫ
}

\author{
Е. В. Зубей
}

\begin{abstract}
Группой Шмидта называют конечную ненильпотентную группу, все собственные подгруппы которой нильпотентны. Группа с нильпотентной максимальной подгруппой, как известно, является разрешимой, если коммутант силовской 2-подгруппы из максимальной подгруппы содержится в центре силовской 2-подгруппы. Если максимальная подгруппа группы ненильпотентна, то в ней существует подгруппа Шмидта. От свойств подгрупп Шмидта из максимальной подгруппы зависит строение самой группы, в частности то, является ли она разрешимой. В данной работе устанавливается разрешимость конечной группы при условии, что некоторые подгруппы Шмидта из максимальной подгруппы группы полунормальны или субнормальны в группе.
\end{abstract}

Ключевые слова: конечная группа, разрешимая группа, подгруппа Шмидта, субнормальная подгруппа, полунормальная подгруппа, максимальная подгруппа.

E. V. Zubei. On the solvability of a finite group with seminormal or subnormal Schmidt subgroups of one of its maximal subgroups.

A Schmidt group is a finite non-nilpotent group all of whose proper subgroups are nilpotent. A group with a nilpotent maximal subgroup is known to be solvable if the derived subgroup of a Sylow 2-subgroup of a maximal subgroup is contained in the center of the Sylow 2-subgroup. If a maximal subgroup of a group is non-nilpotent, then it has a Schmidt subgroup. The structure of a group and, in particular, its solvability, depend on the properties of Schmidt subgroups of its maximal subgroup. In this paper, we establish the solvability of a finite group such that some Schmidt subgroups of its maximal subgroup are seminormal or subnormal in the group.

Keywords: finite group, solvable group, Schmidt subgroup, subnormal subgroup, seminormal subgroup, maximal subgroup.

MSC: MSC20D10, MSC20D20, MSC20D35, MSC20E28

DOI: 10.21538/0134-4889-2019-25-1-55-61

\section{Введение}

Рассматриваются только конечные группы. Используемые обозначения и определения стандартны, их можно найти в $[1 ; 2]$.

Группа $G$ с нильпотентной максимальной подгруппой $M$, как известно, является разрешимой, если коммутант силовской 2-подгруппы $P$ из $M$ содержится в центре подгруппы $P[2$, IV.7.4]. В частности, группа с нильпотентной максимальной подгруппой нечетного порядка разрешима [3]. Эти теоремы нашли отклик во многих работах (см., например, [4-8]).

Если максимальная подгруппа $M$ группы $G$ ненильпотентна, то в $M$ существует подгруппа Шмидта (ненильпотентная группа, у которой все собственные подгруппы нильпотентны). От свойств подгрупп Шмидта из $M$ зависит строение группы $G$, в частности то, является ли группа $G$ разрешимой.

Напомним, что подгруппа $A$ группы $G$ называется полунормальной в $G$, если существует подгруппа $B$ в $G$ такая, что $G=A B$ и $A X$ - собственная в $G$ подгруппа для каждой собственной подгруппы $X$ из $B$. В этой ситуации подгруппу $B$ называют супердобавлением к подгруппе $A$ в $G$. Группы с некоторыми полунормальными подгруппами исследовались в работах многих авторов (см., например, литературу в $[9 ; 10]$ ). Группы с полунормальными 
подгруппами Шмидта изучены В. С. Монаховым и В. Н. Княгиной [11]. Рассматривались также группы, в которых некоторые их силовские или максимальные подгруппы перестановочны с некоторыми подгруппами Шмидта [12-15]. Группы с субнормальными подгруппами Шмидта исследовались в [16-18].

В настоящей работе устанавливается разрешимость группы $G$ при условии, что некоторые подгруппы Шмидта из максимальной подгруппы $M$ группы $G$ полунормальны или субнормальны в $G$. А именно доказываются следующие теоремы.

Теорема 1. Пусть $M-$ максимальная подгруппа конечной группы $G$ и $P$ - силовская 2-подгруппа из $M$. Предположим, что $P^{\prime} \leq Z(P)$ и $M A_{4}$-свободна. Если каждая подгруппа Шмидта из $M$ полунормальна или субнормальна в $G$, то группа $G$ разрешима.

Теорема 2. Пусть $M-$ максимальная подгруппа нечетного индекса конечной группы $G$ и $P$ - силовская 2-подгруппа из $M$. Предположим, что подгруппа $M A_{4}$-свободна и каждая подгруппа Шмидта четного порядка из $M$ полунормальна или субнормальна в $G$. Если $P^{\prime} \leq$ $Z(P)$ или $N_{G}(V) \leq M$ для каждой 2-подгруппы $V$ из $M$, ненормальной в $G$, то $G$ разрешима.

\section{1. Используемые обозначения и результаты}

Ненильпотентная группа, у которой все собственные подгруппы нильпотентны, называется группой Шмидта. Эти группы впервые рассматривались О. Ю. Шмидтом [19], который доказал их бипримарность, нормальность в них одной силовской подгруппы и цикличность другой. В работе [20] приведен обзор результатов о существовании подгрупп Шмидта в конечных группах и их некоторых приложениях в теории классов. Следуя [12;15], условимся называть $S_{\langle p, q\rangle}$-группой группу Шмидта с нормальной силовской $p$-подгруппой и циклической силовской $q$-подгруппой. Подгруппа, являющаяся $\left.S_{\langle p, q\rangle}\right\rangle^{-г р у п п о и ̆, ~ н а з ы в а е т с я ~} S_{\langle p, q\rangle}$-nодгрупnой.

Напомним, что $A^{G}=\left\langle A^{g} \mid g \in G\right\rangle$ - подгруппа, порожденная всеми сопряженными с $A$ подгруппами группы $G$. Запись $N \triangleleft G$ означает, что $N$ - нормальная подгруппа группы $G$. Через $G^{\prime}, Z(G)$ обозначаются коммутант и центр группы $G$ соответственно. Нормализатор подгруппы $A$ в группе $G$ обозначается как $N_{G}(A)$. Полупрямое произведение нормальной в $G$ подгруппы $A$ и подгруппы $B$ записывается так: $G=[A] B$. Группа называется $A_{4}$-свободной, если в ней нет секций, изоморфных $A_{4}$ - знакопеременной группе степени 4.

Лемма 1. (1) Каждая не р-нильпотентная группа $G$ содержит $S_{\langle p, q\rangle}$-подгруппу для некоторого $q \in \pi(G)[2$, IV.5.4].

(2) Каждая не 2-замкнутая группа $G$ содержит $S_{\langle q, 2\rangle}$-подгруппу для некоторого $q \in \pi(G)$ [21, с. 34; 22, следствие 3.1.1].

Лемма 2 [2, теорема IV.7.4]. Пусть $H$ - максимальная подгруппа группь $G$ и $P$ силовскал 2-подгруппа из $H$. Если $H$ нильпотентна и $P^{\prime} \leq Z(P)$, то группа $G$ разрешима.

Лемма 3 [5]. Пусть $P$ - силовскал 2-подгруппа группы $G$. Если $P^{\prime} \leq Z(P)$ и подгруппа $P$ является прямым множителем некоторой максимальной подгруппы группы $G$, то группа $G$ разрешима.

Напомним, что подгруппа $U$ называется субнормальной подгруппой группы $G$, если существуют подгруппы $U_{0}, U_{1}, \cdots, U_{s}$ такие, что $U=U_{0} \triangleleft U_{1} \triangleleft \cdots \triangleleft U_{s-1} \triangleleft U_{s}=G$.

Лемма 4. Пусть $U-$ субнормальная подгруппа группы $G$. Тогда

(1) если $V \leq G$, то $V \cap U$ субнормальна в $V$ [1, лемма 2.41];

(2) если $N \triangleleft G$, то подгруппа $U N / N$ субнормальна в $G$ [1, лемма 2.41];

(3) если $\left\{U_{i} \mid i \in I\right\}$ - некоторое множество субнормальных подгрупп группы $G$, то nодгруппа $U=\left\langle U_{i} \mid i \in I\right\rangle$ субнормальна в $G$ [1, теорема 2.43]. 
Лемма 5. Если $S$ - субнормальная $S_{\langle p, q\rangle}$-подгруппа группљ $G$, то подгруппа $S^{G}$ является p-замкнутой $\{p, q\}$-подгруппой.

Д о к а з а т е л ь с т в о. Пусть $\pi=\{p, q\}$. По [23, следствие 7.7.2(1)] $S \leq O_{\pi}(G)$. Поэтому $S^{G}$ является $\{p, q\}$-подгруппой. Так как $S-q$-нильпотентная подгруппа, то по [23, следствие 7.7.2(3)] $S \leq F_{q}(G)$. Следовательно, $S^{G} \leq F_{q}(G)$. Теперь $S^{G} \leq O_{\pi}(G) \cap F_{q}(G)$. Поэтому $S^{G}$ является $p$-замкнутой $\{p, q\}$-подгруппой.

Здесь $O_{\pi}(G)$ - наибольшая нормальная в $G \pi$-подгруппа, а $F_{q}(G)$ - наибольшая нормальная в $G q$-нильпотентная подгруппа.

Лемма доказана.

Лемма 6. Если в группе $G$ каждая 2-нильпотентная подгруппа Шмидта четного порлдка полунормальна или субнормальна, то $G$ разрешима.

Д о к а з а т е л ь с т в о. Если в группе $G$ нет 2-нильпотентных подгрупп Шмидта четного порядка, то $G 2$-замкнута по п. (2) леммы 1 , а значит и разрешима. Пусть $A-2$-нильпотентная подгруппа Шмидта. Если $A$ полунормальна в $G$, то $A^{G}$ разрешима [11, теорема 1]. Если $A$ субнормальна в $G$, то $A^{G}$ разрешима по лемме 5 .

Пусть $B=\left\langle A^{G}\right| A-2$-нильпотентная подгруппа Шмидта $\rangle$. Тогда $B$ разрешима и нормальна в $G$. Если в фактор-группе $G / B$ нет 2 -нильпотентных подгрупп Шмидта четного порядка, то $G / B$ 2-замкнута по п. (2) леммы 1 и группа $G$ разрешима.

Пусть $K / B-S_{\langle r, 2\rangle}$-подгруппа в $G / B$ для некоторого простого $r$ и $L-$ минимальное добавление к подгруппе $B$ в $K$. По [11, лемма 1] подгруппа $L$ содержит $S_{\langle r, 2\rangle}$-подгруппу $A$ такую, что $A^{L}=L$. Так как $K=L B=A^{L} B \leq A^{G} B \leq B$, то получили противоречие.

Лемма доказана.

\section{2. Признаки разрешимости группы с ограничением на некоторые подгруппы Шмидта}

Теорема 1. Пусть $M-$ максимальная подгруппа конечной группы $G$ и $P-$ силовскал 2-подгруппа из $M$. Предположим, что $P^{\prime} \leq Z(P)$ и $M A_{4}$-свободна. Если кажсдая подгруппа Шмидта из $M$ полунормальна или субнормальна в $G$, то группа $G$ разрешима.

Д о к а з а т е л ь с т в о. Если в $M$ нет подгрупп Шмидта, то $M$ нильпотентна и $G$ разрешима по лемме 2. Поэтому $M$ ненильпотентна и в ней есть подгруппы Шмидта. Пусть $A-$ подгруппа Шмидта из $M$. Предположим, что $A^{G}$ неразрешима. Тогда по лемме 5 подгруппа $A$ не субнормальна в $G$. По условию подгруппа $A$ полунормальна в $G$. Тогда по $[11$, теорема 1$]$ $A$ является 2-замкнутой $\{2,3\}$-подгруппой Шмидта. По свойствам групп Шмидта [22, теорема 1.22] $A / Z(A) \simeq A_{4}$ и подгруппа $M$ не $A_{4}$-свободна; противоречие. Поэтому предположение неверно и $A^{G}$ разрешима. Так как $A$ - произвольная подгруппа Шмидта из $M$, то подгруппа $B=\left\langle A^{G}\right| A-$ подгруппа Шмидта из $\left.M\right\rangle$ нормальна в $G$ и разрешима.

Предположим, что $M B / B$ ненильпотентна. Так как $M B / B \simeq M / M \cap B$, то $M / M \cap B$ ненильпотентна. Пусть $S / M \cap B$ - подгруппа Шмидта из $M / M \cap B$ и $L-$ минимальное добавление к подгруппе $M \cap B$ в $S$. По [11, лемма 1] подгруппа $L$ содержит подгруппу Шмидта $[R] Q$ такую, что $Q$ не содержится в $M \cap B$. Так как $[R] Q \leq L \leq S \leq M$, то $[R] Q \leq B$ по построению подгруппы $B$. Следовательно, $[R] Q \leq M \cap B, Q \leq B$; противоречие. Поэтому допущение неверно и $M B / B$ нильпотентна.

Поскольку $M$ - максимальная в $G$ подгруппа, то либо $M B / B=G / B$, либо $B \leq M$ и $M / B$ - максимальная подгруппа группы $G / B$. Если $M B / B=G / B$, то $G / B$ нильпотентна, а поскольку $B$ разрешима, то $G$ разрешима. 
Пусть $B \leq M$ и $M / B$ - максимальная подгруппа группы $G / B$. Так как $P B / B-$ силовская 2-подгруппа в $M / B$ и

$$
(P B / B)^{\prime}=P^{\prime} B / B \leq Z(P) B / B \leq Z(P B / B),
$$

то $G / B$ разрешима по лемме 2 . Поэтому $G$ разрешима.

Теорема доказана.

Следствие 1. Пусть $M-$ максимальная подгруппа группь $G$ и порлдок $M$ нечетен. Если каждая подгруппа Шмидта из $M$ полунормальна или субнормальна в $G$, то $G$ разрешима.

П р и м е р 1. Условие "максимальная подгруппа $A_{4}$-свободна" в теореме 1 не является лишним. Примером служит группа $G=P S L(2,5)$. В этой группе подгруппа Шмидта $S$, изоморфная группе $A_{4}$, имеет индекс 5 . Поэтому она является максимальной и полунормальной. В подгруппе $S$ силовская 2-подгруппа $P$ абелева, поэтому $P^{\prime} \leq Z(P)$.

П р и м е р 2. Условие " $P^{\prime} \leq Z(P)$ " не является лишним. Примером служит группа $G=P S L(2,17)$. В этой группе силовская 2-подгруппа $P$, изоморфная диэдральной подгруппе порядка 16 , является максимальной и $A_{4}$-свободной. Но $P^{\prime} \leq Z(P)$.

Теорема 2. Пусть $M$ - максимальная подгруппа нечетного индекса конечной группы $G$ и $P$ - силовская 2-подгруппа из $M$. Предположим, что подгруппа $M A_{4}$-свободна и каждая подгруппа Шмидта четного порлдка из $M$ полунормальна или субнормальна в $G$. Если $P^{\prime} \leq$ $Z(P)$ или $N_{G}(V) \leq M$ для каждой 2-подгруппы $V$ из $M$, ненормальной в $G$, то $G$ разрешима.

Д о к а з а т е л ь с т в о. Воспользуемся индукцией по порядку группы $G$. Если в $M$ нет подгрупп Шмидта четного порядка, то $M 2$-разложима по лемме 1 . Если $P^{\prime} \leq Z(P)$, то по лемме 3 группа $G$ разрешима; если $N_{G}(V) \leq M$ для каждой 2-подгруппы $V$ из $M$, ненормальной в $G$, то $G$ разрешима [7, теорема 1]. Следовательно, в $M$ существуют подгруппы Шмидта четного порядка.

Пусть $A$ - подгруппа Шмидта четного порядка из $M$. Предположим, что $A^{G}$ неразрешима. Тогда по лемме 5 подгруппа $A$ не субнормальна в $G$. По условию подгруппа $A$ полунормальна в $G$. Тогда по [11, теорема 1] $A$ является 2 -замкнутой $\{2,3\}$-подгруппой Шмидта. По свойствам групп Шмидта [22, теорема 1.22$] A / Z(A) \simeq A_{4}$ и подгруппа $M$ не $A_{4}$-свободна; противоречие. Поэтому предположение неверно и $A^{G}$ разрешима.

Предположим, что $A^{G} \leq M$ для некоторой подгруппы Шмидта $A$ четного порядка из $M$. Тогда фактор-группа $G / A^{G}$ содержит максимальную подгруппу $M / A^{G}$ и индекс $M / A^{G}$ в группе $G / A^{G}$ нечетен. Ясно, что подгруппа $M / A^{G} A_{4}$-свободна. Пусть $T / A^{G}$ - подгруппа Шмидта четного порядка из $M / A^{G}$ и $L-$ минимальное добавление к $A^{G}$ в $T$, т.е. $T=L A^{G}$. По $[11$, лемма 1] подгруппа $L$ содержит подгруппу Шмидта $[R] Q$ четного порядка такую, что $Q$ не содержится в $A^{G}$ и $L=([R] Q)^{L}=Q^{L}$. Так как

$$
[R] Q \leq L \leq T \leq M,
$$

то $[R] Q$ полунормальна или субнормальна в $G$ по условию теоремы. Если подгруппа $[R] Q$ полунормальна в $G$, то по $[11$, лемма 5$]$ подгруппа $L$ полунормальна в $G$ и по $[11$, лемма 2] подгруппа $L A^{G} / A^{G}=T / A^{G}$ полунормальна в $G / A^{G}$. Если подгруппа $[R] Q$ субнормальна в $G$, то по п. (3) леммы $4 L$ субнормальна в $G$ и по п. (2) леммы 4 подгруппа $L A^{G} / A^{G}=T / A^{G}$ субнормальна в $G / A^{G}$.

Так как $P$ - силовская 2-подгруппа из $M$, то подгруппа $P A^{G} / A^{G}$ является силовской 2-подгруппой в $M / A^{G}$. Если $P^{\prime} \leq Z(P)$, то

$$
\left(P A^{G} / A^{G}\right)^{\prime}=P^{\prime} A^{G} / A^{G} \leq Z(P) A^{G} / A^{G} \leq Z\left(P A^{G} / A^{G}\right) .
$$

Пусть $N_{G}(V) \leq M$ для каждой 2-подгруппы $V$ из $M$, ненормальной в $G$, и $U / A^{G}-2$-подгруппа в $M / A^{G}$, ненормальная в $G / A^{G}$. Тогда $U=U_{2} A^{G}$, где $U_{2}-$ силовская 2-подгруппа 
в $U$. Рассмотрим элемент $x A^{G} \in N_{G / A^{G}}\left(U / A^{G}\right)$. Так как $\left(U / A^{G}\right)^{x A^{G}}=U / A^{G}$, то $U=U^{x}$. По теореме Силова $U_{2}^{x}=U_{2}^{u}$ для $u \in U$. Получаем, что

$$
U^{x}=U_{2}^{x} A^{G}=U_{2}^{u} A^{G}=U .
$$

Итак,

$$
N_{G / A^{G}}\left(U / A^{G}\right)=N_{G}(U) A^{G} / A^{G} \leq M / A^{G} .
$$

Таким образом, для фактор-группы $G / A^{G}$ и ее максимальной подгруппы $M / A^{G}$ все условия доказываемой теоремы выполняются. Так как $A^{G} \neq 1$, то по индукции фактор-группа $G / A^{G}$ разрешима, поэтому $G$ разрешима.

Наконец, рассмотрим случай, когда $A^{G} \nless M$ для каждой подгруппы Шмидта $A$ четного порядка из $M$. Ясно, что $M A^{G}=G$. Из [11, лемма 2] и леммы 4 следует, что каждая подгруппа Шмидта четного порядка из $M$ полунормальна или субнормальна в $M$. По лемме 6 подгруппа $M$ разрешима, поэтому группа $G$ разрешима.

Теорема доказана.

\section{СПИСОК ЛИТЕРАТУРЫ}

1. Монахов В.С. Введение в теорию конечных групп и их классов. Минск: Вышэйшая школа, 2006. $207 \mathrm{c}$.

2. Huppert B. Endliche gruppen I. Berlin; Heidelberg; N Y: Springer, 1967. 796 p. doi: 10.1007/978-3-642-64981-3.

3. Thompson J. Finite groups with fixed point-free automorphisms of prime order // Proc. Nat. Sci., U.S.A. 1959. Vol. 45, no. 4. P. 578-581. doi: 10.1073/pnas.45.4.578.

4. Thompson J. A special class of non-solvable groups // Math. Z. 1960. Vol. 72. P. 458-462. doi: $10.1007 / \mathrm{BF} 01162968$.

5. Белоногов В.А. Один признак разрешимости групп четного порядка // Сиб. мат. журн. 1966. T. 7, № 2. C. $458-459$.

6. Монахов В.С. Некоторые признаки разрешимости групп // Докл. АН БССР. 1970. Т. 14, № 11. C. $986-988$.

7. Монахов В.С. О влиянии свойств максимальных подгрупп на строение конечной группы // Мат. заметки. 1972. Т. 11, № 2. С. 183-190.

8. Baumann B. Endliche nichtauflösbare gruppen mit einer nilpotenten maximal untergruppen // J. Algebra. 1976. Vol. 38. P. 119-135. doi: 10.1016/0021-8693(76)90249-0.

9. Монахов В.С. Конечные группы с полунормальной холловой подгруппой // Мат. заметки. 2006. T. 80 , № 4. C. $573-581$.

10. Guo W. Finite groups with seminormal Sylow subgroups // Acta Mathematica Sinica. 2008. Vol. 24, no. 10. P. 1751-1758. doi: 10.1007/s10114-008-6563-z .

11. Княгина В.Н., Монахов В.С. Конечные группы с полунормальными подгруппами Шмидта // Алгебра и логика. 2007. Т. 46, № 4. С. 448-458.

12. Княгина В.Н. О перестановочности $n$-максимальных подгрупп с $p$-нильпотентными подгруппами Шмидта // Тр. Ин-та математики НАН РБ. 2016. Т. 24, № 1. С. 34-37.

13. Беркович Я. Г., Пальчик Э. М. О перестановочности подгрупп конечной группы // Сиб. мат. журн. 1967. Т. 8, № 4. С. 741-753.

14. Княгина В. Н., Монахов В. С. О перестановочности силовских подгрупп с подгруппами Шмидта // Тр. Ин-та математики и механики УрО РАН. 2010. Т. 16, № 3. С. 130-139.

15. Зубей Е.В., Княгина В.Н., Монахов В.С. О разрешимости конечной группы с $S$-полунормальными подгруппами Шмидта // Укр. мат. журн. 2018. Т. 70, № 11. С. 1511-1518.

16. Княгина В.Н., Монахов В.С. О конечных группах с некоторыми субнормальными подгруппами Шмидта // Сиб. мат. журн. 2004. Т. 45, № 6. С. 1316-1322.

17. Ведерников В.А. Конечные группы с субнормальными подгруппами Шмидта // Алгебра и логика. 2007. Т. 46, № 6. С. 669-687.

18. Al-Sharo Kh. A., Skiba A.N. On finite groups with $\sigma$-subnormal Schmidt subgroups // Commun. Algebra. 2017. Vol. 45, no. 10. P. 4158-4165. doi: 10.1080/00927872.2016.1236938.

19. Шмидт О.Ю. Группы, все подгруппы которых специальные // Мат. сб. 1924. Т. 31. С. 366-372. 
20. Монахов В.С. Подгруппы Шмидта, их существование и некоторые приложения // Тр. Укр. мат. конгресса (2001) / Ин-т математики НАНУ. Киев, 2002. Сек. № 1. С. 81-90.

21. Беркович Я.Г. Теорема о ненильпотентных разрешимых подгруппах конечной группы // Конечные группы / ред. Я. Г. Беркович. Минск: Наука и техника, 1966. С. 24-39. ISBN: 978-5-458-54866-3 .

22. Монахов В.С. О подгруппах Шмидта конечных групп // Вопросы алгебры. 1998. Вып. 13. C. $153-171$.

23. Шеметков Л.А. Формации конечных групп. Минск: Наука, 1978. 271 с.

Поступила 12.12.2018

После доработки 30.01.2019

Принята к публикации 4.02.2019

Зубей Екатерина Владимировна

аспирант

Гомельский государственный университет им. Ф. Скорины

г. Гомель

e-mail: ekaterina.zubey@yandex.ru

\section{REFERENCES}

1. Monakhov V.S. Vvedenie v teoriyu konechnykh grupp $i$ ikh klassov [Introduction to the theory of finite groups and their classes]. Minsk: Vysheishaya Shkola Publ., 2006, 207 p. ISBN: 985-06-1114-6 .

2. Huppert B. Endliche Gruppen I. Berlin; Heidelberg; N Y: Springer, 1967, 796 p. doi: 10.1007/978-3-642-64981-3 .

3. Thompson J. Finite groups with fixed point-free automorphisms of prime order. Proc. Nat. Sci., U.S.A., 1959, vol. 45, no. 4, pp. 578-581. doi: 10.1073/pnas.45.4.578.

4. Thompson J. A special class of non-solvable groups. Math. Z., 1960, vol. 72, pp. 458-462. doi: $10.1007 /$ BF01162968.

5. Belonogov V.A. Solvability criterion for groups of even order. Sib. Math. J., 1966, vol. 7, no. 2, pp. 458-459 (in Russian).

6. Monakhov V.S. Some solvability criteria of groups. DAN BSSR, 1970, vol. 14, no. 11, pp. 986-988 (in Russian).

7. Monakhov V.S. Influence of properties of maximal subgroups on the structure of a finite group. Math. Notes, 1972, vol. 11, no. 2, pp. 115-118. doi: 10.1007/BF01097928.

8. Baumann B. Endliche nichtauflösbare gruppen mit einer nilpotenten maximal untergruppen. J. Algebra, 1976, vol. 38, pp. 119-135. doi: 10.1016/0021-8693(76)90249-0 .

9. Monakhov V.S. Finite groups with a seminormal Hall subgroup. Math. Notes, 2006, vol. 80, no. 4, pp. 542-549. doi: $10.4213 / \mathrm{mzm} 2850$.

10. Guo W. Finite groups with seminormal Sylow subgroups. Acta Mathematica Sinica, 2008, vol. 24, no. 10, pp. 1751-1758. doi: 10.1007/s10114-008-6563-z .

11. Knyagina V.N., Monakhov V.S. Finite groups with seminormal Schmidt subgroups. Algebra and Logic, 2007, vol. 46, no. 4, pp. 244-249. doi: 10.1007/s10469-007-0023-1.

12. Knyagina V.N. On permutability of $n$-maximal subgroups with $p$-nilpotent Schmidt subgroups. Trudy Instituta Matematiki NAN Respubliki Belarus, 2016, vol. 24, no. 1, pp. 34-37 (in Russian).

13. Berkovich Ya.G., Pal'chik, E.M. On the commutability of subgroups of a finite group. Sib. Math. J., 1967, vol. 8, no. 4, pp. 560-568. doi: 10.1007/BF02196475.

14. Knyagina V.N., Monakhov V.S. On the permutability of Sylow subgroups with Schmidt subgroups. Proc. Steklov Institute Math., 2011, vol. 272, suppl. 1, pp. 55-64. doi: 10.1134/S0081543811020052 .

15. Zubei E.V., Knyagina V.N., Monakhov V.S. On the solvability of a finite group with $S$-seminormal Schmidt subgroups. Ukr. Mat. Zhurn., 2018, vol. 70, no. 11, pp. 1511-1518 (in Russian).

16. Knyagina V.N., Monakhov V.S. Finite groups with subnormal schmidt subgroups. Siberian Math. J., 2004, vol. 45, no. 6, pp. 1075-1079. doi: 10.1023/B:SIMJ.0000048922.59466.20.

17. Vedernikov V.A. Finite groups with subnormal Schmidt subgroups. Algebra and Logic, 2007, vol. 46, no. 6, pp. 363-372. doi: 10.1007/S10469-007-0036-9. 
18. Al-Sharo Kh. A., Skiba A.N. On finite groups with $\sigma$-subnormal Schmidt subgroups. Commun. Algebra, 2017, vol. 45, no. 10, pp. 4158-4165. doi: 10.1080/00927872.2016.1236938.

19. Schmidt O. Groups, all subgroups of which are special. Mat. Sb., 1924, vol. 31, no. 3-4, pp. 366-372 (in Russian).

20. Monakhov V.S. The Schmidt subgroups, their existence and some applications. Proc. Ukr. Math. Congr., 2001, Kiev, 2002, sec. 1, pp. 81-90 (in Russian).

21. Berkovich Ya.G. A theorem on non-nilpotent solvable subgroups of a finite group. In: Finite groups, Berkovich Ya.G. (ed.), Minsk, Nauka i Tekhnika Publ., 1966, pp. 24-39 (in Russian). ISBN: 978-5-458-54866-3 .

22. Monakhov V.S. The Schmidt subgroups of finite groups. Voprosy Algebry, 1998, no. 13, pp. 153-171 (in Russian).

23. Shemetkov L.A. Formatsii konechnykh grupp [Formations of finite groups]. Minsk: Nauka Publ., 1978, $271 \mathrm{p}$.

Received December 12, 2018

Revised January 30, 2019

Accepted February 4, 2019

Ekaterina Vladimirovna Zubei, doctoral student, Francisk Skorina Gomel State University, Gomel, 246019, Republic of Belarus, e-mail: ekaterina.zubey@yandex.ru . 